OPEN ACCESS

Edited by:

Morten Ring Eskildsen,

University of Notre Dame,

United States

Reviewed by:

Konrad Jerzy Kapcia,

Adam Mickiewicz University, Poland

Daniel Agterberg,

University of Wisconsin-Milwaukee,

United States

*Correspondence:

Victor D. Lakhno

lak@impb.ru

Specialty section:

This article was submitted to Condensed Matter Physics,

a section of the journal

Frontiers in Physics

Received: 01 February 2021

Accepted: 19 August 2021

Published: 04 October 2021

Citation:

Lakhno VD (2021) Translation-Invariant Bipolarons and Charge Density Waves

in High-

Temperature Superconductors.

Front. Phys. 9:662926.

doi: 10.3389/fphy.2021.662926

\section{Translation-Invariant Bipolarons and Charge Density Waves in High-Temperature Superconductors}

\author{
Victor D. Lakhno * \\ Keldysh Institute of Applied Mathematics of Russian Academy of Sciences, Moscow, Russia
}

A correlation is established between the theories of superconductivity based on the concept of charge density waves (CDWs) and the translation invariant (TI) bipolaron theory. It is shown that CDWs are originated from TI-bipolaron states in the pseudogap phase due to the Kohn anomaly and form a pair density wave (PDW) for wave vectors corresponding to nesting. Emerging in the pseudogap phase, CDWs coexist with superconductivity at temperatures below those of superconducting transition, while their wave amplitudes decrease as a Bose condensate is formed from TI bipolarons, vanishing at zero temperature.

Keywords: paired states, TI-bipolaron mass, Peierls transition, Kohn anomaly, charge density waves

\section{INTRODUCTION}

Presently, there is no agreement about the microscopic nature of the high-temperature superconductivity (HTSC). At the same time, there are phenomenological models such as a Ginzburg-Landau model [1], a model of charge density waves (CDWs) or pair density waves (PDWs) (the recent review on the theory and experiment with CDW/PDW in hightemperature superconductors, ultracold atomic gases, and mesoscopic devices was published by Agterberg et al. [2]) and a model of spin density waves which enable one to describe numerous HTSC experiments [3]. These models are silent on the nature of paired states taking part in SC. In author's works [4-7], paired states mean translation invariant (TI) bipolaron states formed by a strong electron-phonon interaction similar to Cooper pairs (for the review of earlier pioneering works devoted to superconductivity based on the theory of small-radius bipolarons, see [8]). TI bipolarons are plane waves with a small correlation length capable of forming a Bose-Einstein condensate with high transition temperature which possesses SC properties. A correlation between the Bardeen-Cooper-Schrieffer (BCS) theory [9] and the Ginzburg-Landau theory was established by Gor'kov [10]. The aim of this work was to establish a correlation between the TI-bipolaron theory of SC and CDW (PDW).

\section{RESULTS}

\section{General Relations for the Spectrum of a Moving TI Bipolaron}

TI bipolarons are formed at a temperature $T^{*}$ which is much higher than that of an SC transition $T_{c}$ (Refs. [4-7]). For $T_{c}<T<T^{*}$ and in the absence of a Fermi surface with a sharp boundary, an ensemble of TI bipolarons would be an ideal gas whose particles would have a spectrum $s=\left\{v_{k}^{2}(\overrightarrow{\mathcal{P}})\right\}$, determined by the dispersion equation (Refs. [4-7]): 


$$
\begin{aligned}
& 1=\frac{2}{3} \sum_{k} \frac{k^{2}\left|f_{k}\right|^{2} \omega_{k}}{s-\omega_{k}^{2}}, \\
& \omega_{k}=\omega_{0}(\vec{k}, \overrightarrow{\mathcal{P}})-\frac{\vec{k} \overrightarrow{\mathcal{P}}}{M}+\frac{k^{2}}{2 M}-\frac{\vec{k}}{M} \sum_{k^{\prime}} \vec{k}\left|f_{k^{\prime}}\right|^{2},
\end{aligned}
$$

$f_{k}=f_{k}(\vec{k}, \overrightarrow{\mathcal{P}})$ are parameters determining the ground state energy $E_{b p}(\overrightarrow{\mathcal{P}})$ of a TI-bipolaron, $\overrightarrow{\mathcal{P}}$ is the total momentum of a TI bipolaron, $M=2 m$, where $m$ is the mass of a band electron (hole), and $\omega_{0}(\vec{k}, \overrightarrow{\mathcal{P}})$ is the phonon frequency in an electron gas around TI bipolarons.

In describing the Kohn anomaly, one usually proceeds from Fröhlich Hamiltonian of the following form [11]:

$$
H=\sum_{p} \varepsilon(\vec{p}) c_{\vec{p}}^{+} c_{\vec{p}}+\sum_{\vec{p}} \hbar \widetilde{\omega}(k) b_{\vec{k}}^{+} b_{\vec{k}}+\sum_{\vec{p} \vec{k}} g(\vec{k}) c_{\vec{p}+\vec{k}}^{+} c_{\vec{p}}\left(b_{-\vec{k}}^{+}+b_{\vec{k}}\right),
$$

where the first term corresponds to a free electron gas; $c_{p}^{+}, c_{p}$ are the operators of the birth and annihilation of an electron with energy $\varepsilon(\vec{p})$, respectively. The second term corresponds to Hamiltonian of the lattice; $b_{\vec{k}}^{+}, b_{\vec{k}}$ are operators of the birth and annihilation of lattice oscillations with energy $\widetilde{\omega}(k)$, respectively. The third term describes interactions of electrons with the lattice; $g(k)$ is the matrix element of the interaction.

Renormalization of phonon frequencies corresponding to (2) is determined by the expression [11]:

$$
\omega^{2}(\vec{k})=\widetilde{\omega}(\vec{k})^{2}+2 \widetilde{\omega}(\vec{k})|g(\vec{k})|^{2} \operatorname{Re}[\chi(\vec{k})],
$$

where $\omega(\vec{k})$ are phonons renormalized by an interaction with an electron gas whose polarizability is determined by $\chi(\vec{k})$.

The Kohn anomaly describes vanishing of renormalized phonon modes $\omega(k)$ for $k=\mathcal{P}_{C D W}$.

In the TI-bipolaron theory of SC [4-7], bipolarons are believed to be immersed into an electron gas. The properties of such bipolarons are also described by Fröhlich Hamiltonian of the form (2), but with a field of already renormalized phonons with energies $\omega(k)$ and, accordingly, a matrix element of the interaction $V(\vec{k})$ instead of $g(\vec{k})$.

It should be noted that spectral equation $\mathbf{1}$ is independent of the form of $V(\vec{k})$.

The experimental evidence of the occurrence of renormalized phonons with zero energy in layered HTSC cuprates is the absence of a gap in the nodal direction in these materials which, by definition, is the phonon frequency in the TI bipolaron theory of SC.

The wave function of a TI-bipolaron with the wave vector $\overrightarrow{\mathcal{P}}$ will have the following form:

$$
|\Psi(\overrightarrow{\mathcal{P}})\rangle_{b p}=e^{i \overrightarrow{\mathcal{P}} \vec{R}}|\Psi(0)\rangle_{b p},
$$

where $\vec{R}$ comprises coordinates of the bipolaron mass center. The explicit form of the wave function $|\Psi(0)\rangle_{b p}$ with zero momentum is well established [12]. An expression for $\overrightarrow{\mathcal{P}}$ can be derived by calculating the mathematical expectation of the operator of the total momentum $\widehat{\mathcal{P}}$ :

$$
\overrightarrow{\mathcal{P}}=\langle\Psi(\overrightarrow{\mathcal{P}})|\widehat{\mathcal{P}}| \Psi(\overrightarrow{\mathcal{P}})\rangle=M \vec{u}+\sum_{k} \vec{k}\left|f_{k}\right|^{2},
$$

where $\vec{u}$ is the velocity of a TI bipolaron. Assuming that $\overrightarrow{\mathcal{P}}=M_{b p} \vec{u}$, where $M_{b p}$ is the bipolaron mass, we can rewrite (5) and express $M_{b p}$ as follows:

$$
M_{b p}=\frac{M}{1-\eta}, \eta=\frac{\overrightarrow{\mathcal{P}}}{\mathcal{P}^{2}} \sum_{k} \vec{k}\left|f_{k}\right|^{2} .
$$

With the use of (6) expression for $\omega_{k}$ from (1) can be rewritten as follows:

$$
\omega_{k}=\omega_{0}(\vec{k}, \overrightarrow{\mathcal{P}})+\frac{k^{2}}{2 M}-\frac{\vec{k} \overrightarrow{\mathcal{P}}}{M_{b p}} .
$$

It follows from (6) that in the case of a weak and intermediate coupling (when TI-bipolaron states are metastable for $\overrightarrow{\mathcal{P}}=0$ ), the exact form of $f_{k}(\vec{k}, \overrightarrow{\mathcal{P}})$ is known [13], and in the case of bipolaron, it is equal to

$$
f_{k}(\vec{k}, \overrightarrow{\mathcal{P}})=-\frac{V_{k}}{\omega_{0}(\vec{k}, \overrightarrow{\mathcal{P}})-\frac{\vec{k} \overrightarrow{\mathcal{P}}}{2 M}(1-\eta)+\frac{k^{2}}{2 M}},
$$

where $V_{k}$ is the electron-phonon matrix element, and the expression for the TI-bipolaron effective mass will have the simple form: $M_{b p}=$ $M(1+\alpha / 6)$, where $\alpha$ is a constant of the electron-phonon interaction; that is, mass $M_{b p}$ is equal to the sum of masses of individual polarons. For a large $\alpha$, good approximations for $f_{k}$ are available only for $\overrightarrow{\mathcal{P}}=0$. For this reason, the calculation of the effective mass of a TI bipolaron is rather difficult.

\section{TI Bipolarons and Charge Density Waves}

Subject to availability of a Fermi surface with a sharp boundary, the TI-bipolaron gas under consideration will have some peculiarities. Thus, if there are rather large fragments on this surface which can be superimposed by transferring one of them onto vector $\overrightarrow{\mathcal{P}}$, then if the size of these fragments is rather large, the coupling between them will be rather strong, which will lead to the Peierls deformation of the lattice in the direction of this nesting. A loss of energy associated with the lattice deformation will be compensated by a gain in the energy of a bipolaron gas which forms a charge density wave with the wave vector $\overrightarrow{\mathcal{P}}=\overrightarrow{\mathcal{P}}_{C D W}$. The fact of a gain in the energy of a TI bipolaron follows from Equation 1, whose solution leads to the spectrum of a TI-bipolaron $E_{k}(\overrightarrow{\mathcal{P}})$, where

$$
E_{k}(\overrightarrow{\mathcal{P}})=\left\{\begin{array}{l}
E_{b p}(\overrightarrow{\mathcal{P}}), \quad k=0 \\
E_{b p}(\overrightarrow{\mathcal{P}})+\omega_{0}(\overrightarrow{\mathcal{P}}, \vec{k})+\frac{k^{2}}{2 M}-\frac{\vec{k} \overrightarrow{\mathcal{P}}}{M_{b p}}, \quad k \neq 0
\end{array},\right.
$$

that is, $E_{k}=E_{b p}+\omega_{k}$, and $v_{k}=\omega_{k}$ for $k \neq 0$.

The gain in the energy is caused by the above considered Kohn anomaly [14] which implies that for $\mathcal{P}=\mathcal{P}_{C D W}$ (in 1D-metal 


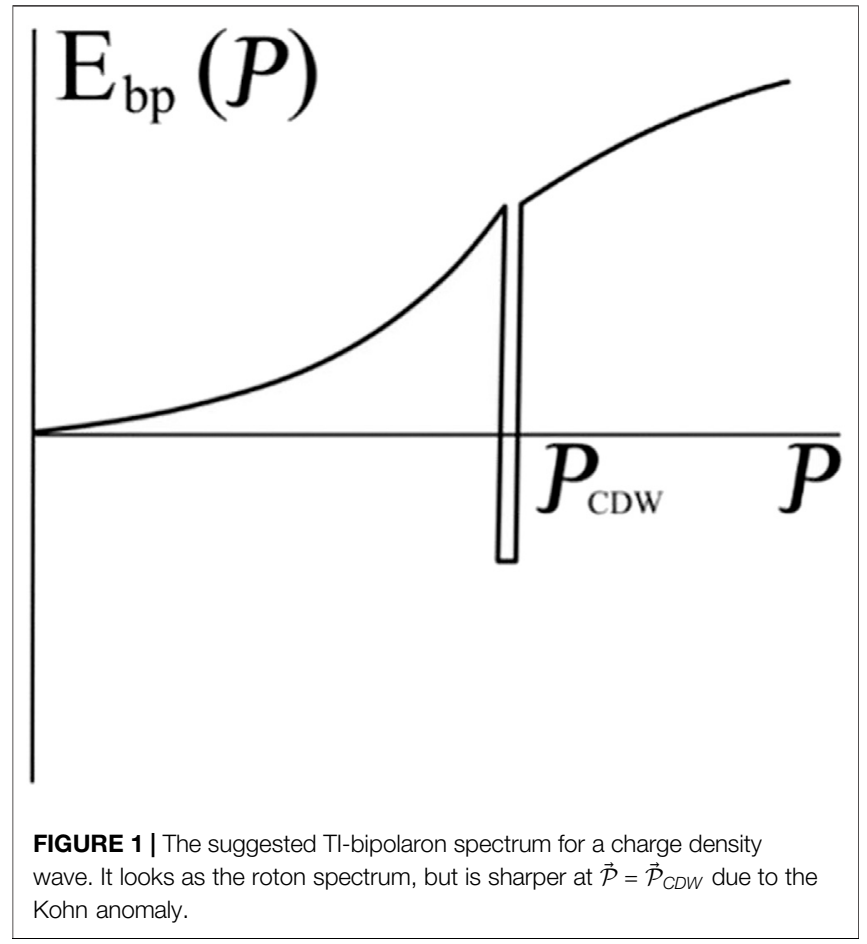

$\mathcal{P}_{C D W}=2 k_{F}, \omega_{0}\left(2 k_{F}\right)=0$, where $k_{F}$ is a Fermi momentum), the phonon frequency $\omega_{0}\left(\mathcal{P}_{C D W}, k\right)=\omega_{C D W}$ softens, and, as a consequence, the energy $E_{b p}$ greatly decreases due to a sharp increase in the constant of the electron-phonon interaction $\alpha \sim 1 / \omega_{C D W}^{1 / 2}$. For $\quad \omega_{C D W} \rightarrow 0, \alpha_{C D W} \rightarrow \infty$, accordingly $M_{b p} \rightarrow \infty$ and CDWs appears to be practically immobile.

The general expression for $E_{b p}(\overrightarrow{\mathcal{P}})$ is complicated, and even in the case of $\overrightarrow{\mathcal{P}}=0$, there are only variational estimates for it [12]. It can be assumed that the general form of the $E_{b p}$ dependence on $\mathcal{P}$ will be $\delta$-shaped, in which the $\delta$-shaped minimum will correspond to $\mathcal{P}=\mathcal{P}_{C D W}$ (Figure 1).

For this spectrum, TI bipolarons will pass on to the state with the energy minimum $E_{b p}\left(\mathcal{P}_{C D W}\right)$ to form a single charge density wave consisting of paired states and determined by expression (4).

Hence, for $T_{C D W}, T_{b p}>T_{C}$, where $T_{C D W}=\mid E_{b p}\left(\mathcal{P}_{C D W}\right)$ $-E_{b p}(0)\left|, \quad T_{b p}=\right| E_{b p}(0)-2 E_{p}(0) \mid, E_{p}(0)$ is the polaron ground state energy, the pseudogap precedes the SC. If in this case the condition $T_{C D W}>T_{b p}$ is fulfilled, then the pseudogap is a coherent pseudophase, and for $T_{b p}>T_{C D W}$, a coherent pseudophase is preceded by a non-coherent phase of free pairs. If the inequality $T_{C D W}, T_{b p}>T_{C}$ is not fulfilled, then the smallest quantity $\left(T_{C D W}\right.$ or $\left.T_{b p}\right)$ becomes equal to $T_{C}$. In any case, the superconducting phase coexists with the pseudogap one which disappears for $T=0$, when all the pairs are in a Bose condensate and the CDW amplitude vanishes.

Energetical advantageousness of a condensate phase follows from expressions (7), (8), which suggest that a homogeneous Bose condensate has a lower energy on condition:

$$
\mathcal{P}<M_{b p} \sqrt{2 \omega_{0}(\mathcal{P}, k) / M} .
$$

It should be noted that the scenario considered is close in many respects to the Fröhlich superconductivity model [15]. In the Fröhlich model, it was assumed that two electrons with opposite momenta (as in BCS) on the Fermi surface are connected by a phonon with the wave vector $\mathcal{P}_{C D W}\left(2 k_{F}\right.$ in $1 \mathrm{D}$ case $)$, thereby forming a charged phonon. Being bosons, such phonons, in a macroscopic number, can be in the same state with the wave vector $\mathcal{P}_{C D W}$ to form a CDW (Fröhlich charge wave). Such a wave, however, will not be superconducting, since pinning which always takes place in real crystals or its scattering by normal carriers will hinder such a wave. The main difference between the TI-bipolaron description and Fröhlich approach is in the formation of a Bose condensate of TI bipolarons (which form a wave of Fröhlich charged phonons), which is just responsible for superconductivity.

\section{DISCUSSION-COMPARISON WITH EXPERIMENT}

To be more specific, let us consider the case of a HTSC such as YBCO. The vector of a CDW in YBCO lies in $a b$-plane and has two equally likely directions: along the $a$-axis $\left(\overrightarrow{\mathcal{P}}_{C D W, x}\right)$ and along the $b$-axis $\left(\overrightarrow{\mathcal{P}}_{C D W, y}\right)$, corresponding to antinodal directions. For $T_{c}<T<T^{*}$, these directions of $\overrightarrow{\mathcal{P}}_{C D W}$ correspond to nonzero soft phonon modes $\omega_{0}\left(\mathcal{P}_{C D W}, k\right)$. The occurrence of a CDW in these directions in $\mathrm{YBCO}$ was detected in a lot of experiments including those on nuclear magnetic resonance [16-18], resonant inelastic X-ray scattering [19-21], resonant scattering, and diffraction of hard X-rays $[22,23]$. Relevant softening of phonon modes in the course of a CDW formation was also observed [24].

Still greater softening of phonon modes can be expected for $T<T_{c}$. A phonon mode corresponding to a nodal direction can vanish, which corresponds to a lack of a gap in the nodal direction. This fact was confirmed experimentally [22] for $\mathrm{Bi}_{2} \mathrm{Sr}_{2-x} \mathrm{La}_{x} \mathrm{CuO} \mathrm{O}_{6+\delta}$ with the use of combined methods of resonant $\mathrm{X}$-ray scattering, scanning tunneling microscopy, and angle-resolved photoelectron spectroscopy. In the case of YBCO with $\omega_{0}\left(\mathcal{P}_{C D W}, k\right)=\Delta_{0}\left|\cos k_{x} a-\cos k_{y} a\right|$ (absolute value of CDW pairing amplitudes or energy gap $[25,26]$ which are the renormalized phonon frequency in a TI-bipolaron theory) according to (9), any value of $\mathcal{P}_{C D W}$ leads to instability of a CDW and the formation of an SC phase in this direction while retaining the pseudogap state in the antinodal direction.

It should be noted that in the approach suggested, a difference between CDWs and PDWs disappears and $\mathcal{P}_{C D W}=\mathcal{P}_{P D W}$ (Ref. [27]).

\section{DATA AVAILABILITY STATEMENT}

The original contributions presented in the study are included in the article/Supplementary Material; further inquiries can be directed to the corresponding author.

\section{AUTHOR CONTRIBUTIONS}

VL contributed to conception and design of the study, wrote the manuscript, read, and approved the submitted version. 


\section{REFERENCES}

1. Larkin A, and Varlamov A. Theory of Fluctuations in Superconductors. Oxford UK: Oxford University Press (2005). doi:10.1093/acprof:oso/ 9780198528159.001.0001

2. Agterberg DF, Séamus Davis JC, Edkins SD, Fradkin E, Van Harlingen DJ, Kivelson SA, et al. The Physics of Pair-Density Waves: Cuprate Superconductors and beyond. Annu Rev Condens Matter Phys (2020) 11: 231-70. doi:10.1146/annurev-conmatphys-031119-050711

3. Grüner G. Density Waves in Solids. Reading: Addison-Wesley (1994). doi:10.1201/9780429501012

4. Lakhno VD. Superconducting Properties of 3D Low-Density TranslationInvariant Bipolaron Gas. Adv Condensed Matter Phys (2018) 2018:1380986. doi:10.1155/2018/1380986

5. Lakhno V. Superconducting Properties of a Nonideal Bipolaron Gas. Physica C: Superconductivity its Appl (2019) 561:1-8. doi:10.1016/j.physc.2018.10.009

6. Lakhno VD. Superconducting Properties of 3D Low-Density TI-Bipolaron Gas in Magnetic Field. Condensed Matter (2019) 4(2):43. doi:10.3390/ condmat 4020043

7. Lakhno VD. Translational-Invariant Bipolarons and Superconductivity. Condensed Matter (2020) 5(2):30. doi:10.3390/condmat5020030

8. Micnas R, Ranninger J, and Robaszkiewicz S. Superconductivity in NarrowBand Systems with Local Nonretarded Attractive Interactions. Rev Mod Phys (1990) 62:113. doi:10.1103/RevModPhys.62.113

9. Bardeen J, Cooper LN, and Schrieffer JR. Theory of Superconductivity. Phys Rev. (1957) 108:1175. doi:10.1103/PhysRev.108.1175

10. Gor'kov LP. Microscopic Derivation of the Ginzburg-Landau Equations in the Theory of Superconductivity. Sov Phys JETP (1959) 9:1364. http://www.jetp. ras.ru/cgi-bin/e/index/e/9/6/p1364?a=list.

11. Grimvall G. The Electron-Phonon Interaction in Metals. ISBN: 9780444861054. Amsterdam: North-Holland Publ. Comp. (1981).

12. Lakhno VD. Pekar's Ansatz and the strong Coupling Problem in Polaron Theory. Phys.-Usp. (2015) 58:295-308. doi:10.3367/UFNe.0185.201503d.0317

13. Lee TD, Low FE, and Pines D. The Motion of Slow Electrons in a Polar crystal. Phys Rev (1953) 90:297. doi:10.1103/PhysRev.90.297

14. Kohn W. Image of the Fermi Surface in the Vibration Spectrum of a Metal. Phys Rev Lett. (1959) 2:393. doi:10.1103/PhysRevLett.2.393

15. Fröhlich H. On the Theory of Superconductivity: the One-Dimensional Case. Proc R Soc Lond A (1954) 223:296-305. doi:10.1098/rspa.1954.0116

16. Wu T, Mayaffre H, Krämer S, Horvatić M, Berthier C, Hardy WN, et al. Magneticfield-induced Charge-Stripe Order in the High-Temperature Superconductor YBa2Cu3Oy. Nature (2011) 477:191-4. doi:10.1038/nature10345

17. Wu T, Mayaffre H, Krämer S, Horvatić M, Berthier C, Kuhns PL, et al. Emergence of Charge Order from the Vortex State of a High-Temperature Superconductor. Nat Commun (2013) 4:2113. doi:10.1038/ncomms3113

18. Wu T, Mayaffre H, Krämer S, Horvatić M, Berthier C, Hardy WN, et al. Incipient Charge Order Observed by NMR in the normal State of YBa2Cu3Oy. Nat Commun (2015) 6:6438. doi:10.1038/ncomms7438
19. Ghiringhelli G, Le Tacon M, Minola M, Blanco-Canosa S, Mazzoli C, Brookes NB, et al. Long-Range Incommensurate Charge Fluctuations in (Y,Nd)Ba2Cu3O6+x. Science 337(6096), 821-5. (2012). doi:10.1126/ science. 1223532

20. Achkar AJ, Sutarto R, Mao X, He F, Frano A, Blanco-Canosa S, et al. Distinct Charge Orders in the Planes and Chains of Ortho-III-Ordered $\mathrm{YBa} 2 \mathrm{Cu} 3 \mathrm{O} 6+\delta$ Super Conductors Identified by Resonant Elastic X-ray Scattering. Phys Rev Lett 109, 167001 (2012). doi:10.1103/PhysRevLett.109.167001

21. Blackburn E, Chang J, Hücker M, Holmes AT, Christensen NB, Liang R, et al. X-Ray Diffraction Observations of a Charge-Density-Wave Order in Superconducting Ortho-II YBa2Cu3O6.54 Single Crystals in Zero Magnetic Field. Phys Rev Lett 110, 137004 (2013). doi:10.1103/ PhysRevLett.110.137004

22. Comin R, Frano A, Yee MM, Yoshida Y, Eisaki H, Schierle E, et al. Charge Order Driven by Fermi-Arc Instability in Bi2Sr2-xLaxCuO6+ $\delta$. Science 343 (6169), 390-2. (2014). doi:10.1126/science.1242996

23. Chang J, Blackburn E, Holmes AT, Christensen NB, Larsen J, Mesot J, et al. Direct Observation of Competition between Superconductivity and Charge Density Wave Order in YBa2Cu3O6.67. Nat Phys (2012) 8:871-6. doi:10.1038/nphys2456

24. Le Tacon M, Bosak A, Souliou SM, Dellea G, Loew T, Heid R, et al. Inelastic $\mathrm{X}$-ray Scattering in YBa2Cu3O6.6 Reveals Giant Phonon Anomalies and Elastic central Peak Due to Charge-Density-Wave Formation. Nat Phys (2014) 10:52-8. doi:10.1038/nphys2805

25. Comin R, Sutarto R, He F, da Silva Neto EH, Chauviere L, Fraño A, et al. Symmetry of Charge Order in Cuprates. Nat Mater (2015) 14:796-800. doi:10.1038/nmat4295

26. Chowdhury D, and Sachdev S. Density-wave Instabilities of Fractionalized Fermi Liquids. Phys Rev B 90, 245136 (2014). doi:10.1103/ PhysRevB.90.245136

27. Hamidian MH, Edkins SD, Joo SH, Kostin A, Eisaki H, Uchida S, et al. Detection of a Cooper-pair Density Wave in $\mathrm{Bi} 2 \mathrm{Sr} 2 \mathrm{CaCu} 2 \mathrm{O} 8+\mathrm{x}$. Nature (2016) 532:343-7. doi:10.1038/nature17411

Conflict of Interest: The author declares that the research was conducted in the absence of any commercial or financial relationships that could be construed as a potential conflict of interest.

Publisher's Note: All claims expressed in this article are solely those of the authors and do not necessarily represent those of their affiliated organizations, or those of the publisher, the editors, and the reviewers. Any product that may be evaluated in this article, or claim that may be made by its manufacturer, is not guaranteed or endorsed by the publisher.

Copyright (c) 2021 Lakhno. This is an open-access article distributed under the terms of the Creative Commons Attribution License (CC BY). The use, distribution or reproduction in other forums is permitted, provided the original author(s) and the copyright owner(s) are credited and that the original publication in this journal is cited, in accordance with accepted academic practice. No use, distribution or reproduction is permitted which does not comply with these terms. 